\title{
COMPLETENESS OF SECOND-ORDER PROPOSITIONAL S4 AND H IN TOPOLOGICAL SEMANTICS
}

\author{
PHILIP KREMER \\ Department of Philosophy, University of Toronto
}

\begin{abstract}
We add propositional quantifiers to the propositional modal logic S4 and to the propositional intuitionistic logic $\mathrm{H}$, introducing axiom schemes that are the natural analogs to axiom schemes typically used for first-order quantifiers in classical and intuitionistic logic. We show that the resulting logics are sound and complete for a topological semantics extending, in a natural way, the topological semantics for $\mathrm{S} 4$ and for $\mathrm{H}$.
\end{abstract}

In this article, we add propositional quantifiers to the propositional modal [intuitionistic] logic $\mathrm{S} 4[\mathrm{H}]$, with axiom schemes that are the natural analogs to axiom schemes typically used for first-order quantifiers in classical logic. We show that the resulting logics are sound and complete for a topological semantics extending, in a natural way, the topological semantics for S4 and for $\mathrm{H}^{1}{ }^{1}$ In particular, we interpret the second-order propositional modal and intuitionistic languages in topological structures, where a topological structure is an ordered pair $\langle X, D\rangle$, with $X$ a topological space and $D \subseteq \mathcal{P}(X)$ a domain over which the propositional quantifiers range. (Extra conditions on $D$ typically have to be met: see below.)

Analogous work has already been done for Kripke semantics: for the modal case, see [1]; and for the intuitionistic case see [2] and [12] and a short discussion in [13]. There is a notable disanalogy between Kripke and topological semantics: in the Kripke semantics for second-order propositional $\mathrm{S} 4$ and $\mathrm{H}$, if we have a constant domain for the quantifiers then, in the modal case, we have to add a Barcan axiom scheme, $\mathbf{B},(\forall p \square A \rightarrow \square \forall p A)$; and, in the intuitionistic case, a constant-domain axiom scheme, $\mathbf{C}, \forall p(A \vee B) \rightarrow(A \vee \forall p B)(p$ is not free in $A$ ). To get completeness in the Kripke semantics for second-order propositional $\mathrm{S} 4[\mathrm{H}]$ without $\mathbf{B}[\mathbf{C}]$, we have to allow varying domains. ${ }^{2}$ In the topological semantics, by contrast, we get completeness for second-order propositional S4 $[\mathrm{H}]$ without $\mathbf{B}[\mathbf{C}]$, even though our topological structures have a constant domain. This is analogous to the firstorder case. Let QS4 [QH] be first-order S4 $[\mathrm{H}]$ without identity, with standard first-order axiom schemes for the quantifiers. In the Kripke semantics we get the completeness of QS4 + a Barcan scheme $[\mathrm{QH}+$ a constant-domain scheme] in a constant-domain semantics, and we only get the completeness of QS4 $[\mathrm{QH}]$ if we allow varying domains. Meanwhile, in the topological semantics we get the completeness of QS4 [QH] with constant domains.

Received: October 19, 2016.

2010 Mathematics Subject Classification: 03B45, 03B20.

Key words and phrases: second-order propositional logic, modal logic, intuitionistic logic, topological semantics.

1 Thanks to Dmitrij Skvortsov for bringing this topic to my attention.

2 See [1] for second-order propositional S4. [2] proves Kripke completeness for simple versions of constant-domain second-order propositional H. [12] generalizes the results from [2] and also proves completeness for variable-domain second-order propositional $\mathrm{H}$. 
Another notable disanalogy between Kripke and topological semantics is that the completeness proofs in the Kripke semantics for second-order propositional $\mathrm{S} 4[\mathrm{H}]$ deliver strong completeness, the claim that every consistent set of formulas is satisfiable, since these proofs use the now standard canonical-models method ([10]). Here we adapt the algebraic completeness methods from [11], which do not seem well-suited to proving strong completeness. ${ }^{3}$ We leave it as an open question whether the second-order propositional logics given below are strongly complete in the topological semantics.

One last remark, before we get down to work. For propositional S4 [H], topological semantics can be seen as a generalization of Kripke semantics. But even more general is the algebraic semantics (see [11]): in the modal case, formulas are interpreted as points in an interior algebra, and in the intuitionistic case, as points in a Heyting algebra. A natural extension of the algebraic semantics to propositionally quantified languages is to interpret these languages in complete interior or Heyting algebras: the completeness of the algebra ensures that quantified formulas can be interpreted. As noted in [14], the completeness proof presented in [3] for the intuitionistic case has a subtle flaw. To our knowledge the question remains open whether a suitable propositionally quantified version of S4 or $\mathrm{H}$ is complete for complete interior or Heyting algebras.

\section{§1. Preliminaries.}

1.1. Syntax. Let $\mathcal{L}$ be a second-order propositional language with a countable set $P V$ of propositional variables; connectives $\&, \vee, \rightarrow$, and $\sim$; and propositional quantifiers $\forall$ and $\exists$. And let $\mathcal{L}^{\square}$ be $\mathcal{L}$ extended with a modal operator $\square$. In either language, $(A \leftrightarrow B)$ is defined as $(A \rightarrow B) \&(B \rightarrow A)$. We use upper case $A, B$, etc., to range over formulas and lower case $p, q$, etc., to range over PV. We let Form be the set of formulas of $\mathcal{L}$ and Form ${ }^{\square}$ be the set of formulas of $\mathcal{L}^{\square}$.

If $p$ and $q$ are propositional variables occurring in $A$, then an occurrence $p$ is in the scope of $q$ if that occurrence of $p$ is in the scope of a quantifier phrase $\forall q$ or $\exists q$. In either language, if $A$ and $B$ are formulas and $p \in \mathrm{PV}$, then $B$ is substitutible for $p$ in $A$ if no free occurrence of $p$ in $A$ is in the scope of a variable with a free occurrence in $B$. If $B$ is substitutible for $p$ in $A$, then we define $A[B / p]$ as the formula that results from replacing every free occurrence of $p$ with $B$-otherwise $A[B / p]$ is undefined. When $A[B / p]$ is defined, we say that it is a substitution instance of $A$.

It will be useful to generalize this last definition in two ways. First, we want a notion of simultaneous substitution of formulas for propositional variables. Second, we want to be able to substitute $B$ for $p$ in $A$, even when $B$ is not substitutible for $p$ in $A$ : for this, we change bound variables. To be precise, suppose that $\varphi: \mathrm{PV} \rightarrow$ Form $^{\square}$. We will extend $\varphi$ to Form $\square$ (and thus also to Form) so that $\varphi(A)$ is, up to changes in bound variables, the result of simultaneously substituting the $\varphi(p)$ 's for the $p$ 's in $A$. First, for any $\varphi: \mathrm{PV} \rightarrow$ Form $^{\square}$, any $B \in$ Form $^{\square}$ and any $p \in \mathrm{PV}$, let $\varphi_{p}^{B}$ be just like $\varphi$ except that

3 This probably explains why, putting second-order propositional logic aside, the issue of strong completeness has not been much addressed in topological semantics. Recently, however, [8] proves the strong completeness of S4 for any dense-in-itself metric space and [9] proves the strong completeness of QS4 for the rational line (with a constant domain for the quantifiers). These articles piggyback on strong completeness in the Kripke semantics, simulating Kripke frames the topological semantics. The strong completeness results in [8] and [9] also apply to $\mathrm{H}$ and to $\mathrm{QH}$. 
$\varphi_{p}^{B}(p)=B$. Now extend each $\varphi: \mathrm{PV} \rightarrow$ Form $\square$ as follows: $\varphi(\sim A)=\sim \varphi(A) ; \varphi(\square A)=$ $\square \varphi(A) ; \varphi(A \& B)=\varphi(A) \& \varphi(B)$ and similarly for $\vee$ and $\rightarrow ; \varphi(\forall p A)=\forall q \varphi_{p}^{q}(A)$, where $q \in \mathrm{PV}$ is chosen so that $q$ does not occur in $A$ and is not free in $\varphi(r)$ for any propositional variable $r$ occurring in $A$; and similarly for $\exists$. Note that, if $\varphi:$ PV $\rightarrow$ Form, the $\varphi(A) \in$ Form for every $A \in$ Form.

Let $l: \mathrm{PV} \rightarrow$ Form $^{\square}$ be the identity function. Also, say that $A$ and $A^{\prime}$ are alphabetical variants, in symbols $A \stackrel{\text { alpha }}{=} A^{\prime}$, iff they differ only in the choice of bound variables. Note that $A \stackrel{\text { alpha }}{=} l(A)$; also, for any $A, B \in$ Form $^{\square}$ and $p \in P V$, there is an $A^{\prime} \in$ Form $^{\square}$ such that $A \stackrel{\text { alpha }}{=} A^{\prime}$ and $B$ is substitutible for $p$ in $A^{\prime}$ and $A^{\prime}[B / p] \stackrel{\text { alpha }}{=} l_{p}^{B}(A)$. Also note the following, where $\varphi: \mathrm{PV} \rightarrow$ Form $^{\square} ; A, B \in$ Form $^{\square} ; p, q \in \mathrm{PV} ; q$ does not occur in $A$; and $q$ is not free in $\varphi(r)$ for any propositional variable $r$ occurring in $A$ :

$$
\begin{array}{rll}
\imath_{q}^{B}\left(\varphi_{p}^{q}(A)\right) & \stackrel{\text { alpha }}{=} & \varphi_{p}^{B}(A) \\
\forall p A & \stackrel{\text { alpha }}{=} \forall q \imath_{p}^{q}(A) \\
\exists p A & \stackrel{\text { alpha }}{=} \exists q \imath_{p}^{q}(A) \\
\varphi(\forall p A) & \stackrel{\text { alpha }}{=} \forall q \varphi\left(l_{p}^{q}(A)\right) \\
\varphi(\exists p A) & \stackrel{\text { alpha }}{=} \exists q \varphi\left(\imath_{p}^{q}(A)\right) .
\end{array}
$$

1.2. Axiomatizations. We define propositionally quantified logics by adding axiom schemes and rules for propositional quantifiers to the base propositional logic, whether $\mathrm{H}$ or S4. Consider the following axiom schemes governing the propositional quantifiers, where $F$ is a subset of Form ${ }^{\square}$ or of Form (depending on the language we are working with):

1. $\forall p(A \rightarrow B) \rightarrow(A \rightarrow \forall p B)$, if $p$ is not free in $A$

2. $\forall p(A \rightarrow B) \rightarrow(\exists p A \rightarrow B)$, if $p$ is not free in $B$

3. $\forall p A \rightarrow A[B / p], B \in \mathrm{F}$ and $B$ is substitutible for $p$ in $A$

4. $A[B / p] \rightarrow \exists p A, B \in \mathrm{F}$ and $B$ is substitutible for $p$ in $A$.

Propositionally quantified intuitionistic logics. For any subset $\mathrm{F}$ of Form let $\mathrm{H}_{\mathrm{F}}^{2}$ be the logic determined in the language $\mathcal{L}$ by all substitution instances of the theorems of $\mathrm{H}$, Axiom Schemes (1)-(4), and the rules, Modus Ponens and Universal Generalization (from $A$ to infer $\forall p A$ ).

Propositionally quantified modal logics. For any subset $F$ of Form $\square$ let $S 4_{\mathrm{F}}^{2}$ be the logic determined in the language $\mathcal{L}^{\square}$ by all substitution instances of the theorems of S4, Axiom Schemes (1)-(4), and the rules, Modus Ponens, Universal Generalization, and Necessitation (from $A$ to infer $\square A$ ).

We will not consider $\mathrm{S}_{\mathrm{F}}^{2}\left[\mathrm{H}_{\mathrm{F}}^{2}\right]$ for arbitrary $\mathrm{F}$. Say that $\mathrm{F}$ is suitable iff $\mathrm{PV} \subseteq \mathrm{F}, \mathrm{F}$ is closed under alphabetic variants, and $\mathrm{F}$ is closed under substitutions: if $\varphi: P V \rightarrow \mathrm{F}$ and $A \in \mathrm{F}$, then $\varphi(A) \in \mathrm{F}$. Many interesting sets of formulas are suitable, for example, the set $P V$ of propositional variables; the set Form ${ }^{\square}$ of formulas of $\mathcal{L}^{\square}$; the set Form of 
formulas of $\mathcal{L}$; the set $F_{\forall \ni}$ of quantifier-free formulas of Form ${ }^{\square}$; and the set $F_{\square \forall \ni}$ of $\square$ and quantifier-free formulas of Form ${ }^{\square}$ ( $F_{\square \forall \ngtr 7}$ is also a subset of Form).

LEMMA 1.1. If $\mathrm{F}$ is suitable and if $A$ and $A^{\prime}$ are alphabetic variants in the language Form ${ }^{\square}$ [Form], then $\left(A \leftrightarrow A^{\prime}\right) \in \mathrm{S} 4_{\mathrm{F}}^{2}\left[\left(A \leftrightarrow A^{\prime}\right) \in \mathrm{H}_{\mathrm{F}}^{2}\right]$.

COROLlaRY 1.2. If $\mathrm{F} \subseteq$ Form $^{\square}[\mathrm{F} \subseteq$ Form] is suitable, $A, B \in \mathrm{F}$ and $p \in \mathrm{PV}$, then the following are theorems of $\mathrm{S}_{\mathrm{F}}^{2}\left[\mathrm{H}_{\mathrm{F}}^{2}\right]: \forall p A \rightarrow \imath_{p}^{B}(A)$ and $\imath_{p}^{B}(A) \rightarrow \exists p A$.

Henceforth, we always assume that $F$ is suitable. If $\mathrm{L}=\mathrm{S} 4_{\mathrm{F}}^{2}\left[\mathrm{H}_{\mathrm{F}}^{2}\right]$ for some $\mathrm{F}$, then we let Form $_{\mathrm{L}}=$ Form $^{\square}$ [Form].

1.3. Semantics. A (topological) structure is an ordered pair $\langle X, D\rangle$ where $X$ is a topological space and $D \subseteq \mathcal{P}(X)$. The structure is intuitionistic iff $D \subseteq \mathcal{O}(X)$, where $\mathcal{O}(X)$ is the set of open sets in $X$. Given a structure $\langle X, D\rangle$, a valuation on $\langle X, D\rangle$ is a function $V: \mathrm{PV} \rightarrow D$. Given a valuation $V, p \in \mathrm{PV}$ and $P \in D$, we define $V[P / p]$ to be the valuation just like $V$ except that it assigns $P$ to $p$. A (topological) model is an ordered triple $M=\langle X, D, V\rangle$, where $\langle X, D\rangle$ is a structure and $V$ is a valuation on $\langle X, D\rangle$. A model $\langle X, D, V\rangle$ is intuitionistic iff $\langle X, D\rangle$ is intuitionistic.

Given a structure $\langle X, D\rangle$, we will extend every valuation $V$ to two functions:

- $V_{\text {mod }}:$ Form $^{\square} \rightarrow \mathcal{P}(X)$, which gives the modal value of each formula in Form ${ }^{\square}$; and

- $V_{\text {int }}:$ Form $\rightarrow \mathcal{O}(X)$, which gives the intuitionistic value of each formula in Form.

First, we define two operations on subsets of $X$, where $I n t_{X}$ is topological interior:

$$
\begin{array}{rll}
\sim_{X} S & =_{\mathrm{df}} \quad \operatorname{Int}_{X}(X-S) \\
S \Rightarrow_{X} S^{\prime} & =_{\mathrm{df}} \quad \operatorname{Int}_{X}\left((X-S) \cup S^{\prime}\right) .
\end{array}
$$

$V_{\text {mod }}$ and $V_{\text {int }}$ are defined as follows:

$$
\begin{aligned}
& V_{\text {mod }}(p)=V(p) \\
& V_{\text {mod }}(A \& B)=V_{\text {mod }}(A) \cap V_{\text {mod }}(B) \\
& V_{\text {mod }}(A \vee B)=V_{\text {mod }}(A) \cup V_{\bmod }(B) \\
& V_{\text {mod }}(A \rightarrow B)=\left(X-V_{\text {mod }}(A)\right) \cup V_{\text {mod }}(B) \\
& V_{\text {mod }}(\sim A)=X-V_{\text {mod }}(A) \\
& V_{\text {mod }}(\square A)=\operatorname{Int}\left(V_{\bmod }(A)\right) \\
& V_{\text {mod }}(\forall p A)=\bigcap_{P \in D} V[P / p]_{\bmod } A \\
& V_{\bmod }(\exists p A)=\bigcup_{P \in D} V[P / p]_{\bmod } A \\
& V_{\text {int }}(p)=V(p) \\
& V_{\text {int }}(A \& B)=V_{\text {int }}(A) \cap V_{\text {int }}(B) \\
& V_{\text {int }}(A \vee B)=V_{\text {int }}(A) \cup V_{\text {int }}(B) \\
& V_{\text {int }}(A \rightarrow B)=V_{\text {int }}(A) \Rightarrow_{X} V_{\text {int }}(B) \\
& V_{\text {int }}(\sim A)=\sim_{X} V_{\text {int }}(A) \\
& V_{\text {int }}(\forall p A)=\operatorname{Int}_{X}\left(\bigcap_{P \in D} V[P / p]_{\text {int }} A\right) \\
& V_{\text {int }}(\exists p A)=\bigcup_{P \in D} V[P / p]_{\text {int }} A \text {. }
\end{aligned}
$$

If $\mathrm{L}=\mathrm{S} 4_{\mathrm{F}}^{2}\left[\mathrm{H}_{\mathrm{F}}^{2}\right]$ for some $\mathrm{F}$, then we let $V_{\mathrm{L}}=V_{\text {mod }}\left[V_{\text {int }}\right]$.

If $M=\langle X, D, V\rangle, \mathrm{L}$ is one of our logics, and $A \in$ Form $_{\mathrm{L}}$, then we say that $M \vDash_{\mathrm{L}} A$ iff $V_{\mathrm{L}}(A)=X$. We say that $\langle X, D\rangle \vDash_{\mathrm{L}} A$ iff $\langle X, D, V\rangle \vDash_{\mathrm{L}} A$ for all valuations $V$ on $\langle X, D\rangle$. 
If $\mathcal{S}$ is a class of structures, then we say that $\mathcal{S} \vDash_{\mathrm{L}} A$ iff $\langle X, D\rangle \vDash_{\mathrm{L}} A$ for every $\langle X, D\rangle \in \mathcal{S}$. Say that $\mathrm{L}$ is sound for a class $\mathcal{S}$ of structures iff, for every $A \in$ Form $_{\mathrm{L}}$, if $A \in \mathrm{L}$ then $\mathcal{S} \vDash_{\mathrm{L}} A$; and that $\mathrm{L}$ is complete for a class $\mathcal{S}$ of structures iff, for every $A \in$ Form $_{\mathrm{L}}$, if $\mathcal{S} \vDash_{\mathrm{L}} A$ then $A \in \mathrm{L}$.

A topological structure $\langle X, D\rangle$ is modally closed under $\mathrm{F} \subseteq$ Form $^{\square}$ iff $V_{\bmod }(A) \in$ $D$, for every valuation $V$ on $\langle X, D\rangle$ and every $A \in \mathrm{F}$. And an intuitionistic topological structure $\langle X, D\rangle$ is intuitionistically closed under $\mathrm{F} \subseteq$ Form iff $V_{\text {int }}(A) \in D$, for every valuation $V$ on $\langle X, D\rangle$ and every $A \in \mathrm{F}$.

THEOREM 1.3. $\mathrm{S}_{\mathrm{F}}^{2}\left[\mathrm{H}_{\mathrm{F}}^{2}\right]$ is sound and complete for the class of all structures modally [intuitionistically] closed under $\mathrm{F}$.

Soundness is routine, and we leave it to the reader. For completeness, see $\S 2$ and $\S 3$.

REMARK 1.4. One concern about Theorem 1.3 is that the condition placed on the structure $\langle X, D\rangle$-namely that it be modally or intuitionistically closed under $\mathrm{F}$-is stated with reference to formulas and to valuations. It would be more appealing to have closure conditions that are given in terms more intrinsic to $\langle X, D\rangle$. For a number of $\mathrm{F}$ 's, we can accomplish this. Say that a topological structure $\langle X, D\rangle$ Boolean-closed iff $D$ is closed under intersection, union, and complementation (wrt $X)$; is interior-closed if $D$ is both Boolean-closed and closed under interiors; and is Heyting-closed iff $D \subseteq \mathcal{O}(X)$ and $D$ is closed under intersection, union, and the operations $\sim_{X}$ and $\Rightarrow_{X}$ defined above. Note the following, where the sets $F_{\forall \ni}$ and $F_{\square \forall 尹}$ are as defined on page 510:

1. Every structure is modally closed under PV.

2. A structure is modally closed under $F_{\square \forall \ngtr}$ iff it is Boolean-closed.

3. A structure is modally closed under $F_{\forall \exists \dashv}$ iff it is interior-closed.

4. Every intuitionistic structure is intuitionistically closed under PV.

5. An intuitionistic structure is intuitionistically closed under $F_{\square \forall 尹}$ iff it is Heytingclosed.

Thus, Theorem 1.3 implies

1. $\mathrm{S} 4 \mathrm{PV}$ is sound and complete for the class of all structures.

2. $S 4_{F_{\square \bigvee \ngtr \nexists}}^{2}$ is sound and complete for the class of Boolean-closed structures.

3. $\mathrm{S} 4_{\mathrm{F}_{y \neq}}^{2}$ is sound and complete for the class of interior-closed structures.

4. $\mathrm{H}_{\mathrm{PV}}^{2}$ is sound and complete for the class of all intuitionistic structures.

5. $\mathrm{H}_{\mathrm{F}_{\square \bigvee \nexists}}^{2}$ is sound and complete for the class of Heyting-closed intuitionistic structures.

In the Appendix, we give intrinsic conditions on a topological structure equivalent to that structure being closed under Form ${ }^{\square}$ : a similar strategy gives intrinsic conditions on an intuitionistic topological structure equivalent to that structure being intuitionistically closed under Form.

REMARK 1.5. Say that a model $\langle X, D, V\rangle$ is modally principal if $D=\mathcal{P}(X)$ and is intuitionistically principal iff $D=\mathcal{O}(X)$. Let $S 4_{+}^{2}={ }_{\mathrm{df}}\left\{A \in \mathrm{Form}^{\square}: V_{\bmod }(A)=X\right.$ for every modally principal model $\langle X, D, V\rangle\}$ and $\mathrm{H}_{+}^{2}={ }_{\mathrm{df}}\left\{A \in\right.$ Form : $V_{\text {int }}(A)=X$ for every intuitionistically principal model $\langle X, D, V\rangle\}$. [7] shows that second-order arithmetic can be recursively embedded in $\mathrm{S} 4_{+}^{2}$, and claims without proof that second-order arithmetic can be recursively embedded in $\mathrm{H}_{+}^{2}$. This is related to earlier work in Kripke semantics: 
the principal second-order propositional extensions of the modal logics $\mathrm{S} 4, \mathrm{~S} 4.2, \mathrm{~K} 4, \mathrm{~K}, \mathrm{~T}$ and $\mathrm{B}$ and of the intuitionistic logic $\mathrm{H}$ are all recursively isomorphic to full second-order logic. ${ }^{4}$

§2. Completeness of $S 4_{\mathrm{F}}^{2}$. Fix a suitable $\mathrm{F}$. We will freely use Lemma 1.1, which tells us that $S 4_{\mathrm{F}}^{2}$ is closed under alphabetic variants. The completeness proof for $\mathrm{S} 4 \mathrm{~F}_{\mathrm{F}}^{2}$ relies on our ability to embed the Lindenbaum algebra of $S 4_{F}^{2}$ in the algebra of sets of some topological space: this is exactly the strategy used in [11] for propositional and first-order modal and intuitionistic logic. Now for the details.

An interior algebra is a seven-tuple $\mathbf{A}=\langle\mathbf{A}, \sqcap, \sqcup,-, 0,1, \mathbf{I}\rangle$, where $\langle\mathbf{A}, \sqcap, \sqcup,-, 0,1\rangle$ is a Boolean algebra and $\mathbf{I}: \mathbf{A} \rightarrow \mathbf{A}$ satisfies the following, for any $a, b \in \mathbf{A}: \mathbf{I} a \leq a$, $\mathbf{I I} a=\mathbf{I} a, \mathbf{I}(a \sqcap b)=\mathbf{I} a \sqcap \mathbf{I} b, \mathbf{I} 1=1$ : here, and below, $a \leq b$ is the partial order defined as $a \sqcup b=b$. $\mathbf{A}$ is degenerate if $0=1$. Otherwise $\mathbf{A}$ is nondegenerate.

One useful example is the algebra of subsets of a topological space. If $X$ is a topological space, then define $\mathbf{A}_{X}={ }_{\mathrm{df}}\left\langle\mathcal{P}(X), \cap, \cup,-_{X}, \emptyset, X\right.$, Int $\left._{X}\right\rangle$ : here $-_{X}$ is complementation relative to $X\left(-{ }_{X}(S)=(X-S)\right.$, for $\left.S \subseteq X\right)$ and $I n t_{X}$ is topological interior. Note that this is an interior algebra.

Another useful example of an interior algebra is the Lindenbaum algebra for $\mathrm{S} 4{ }_{\mathrm{F}}^{2}$. Say that any two formulas $A$ and $B$ are $\mathrm{S} 4 \mathrm{~F}_{\mathrm{F}}^{2}$-equivalent iff $(A \leftrightarrow B) \in \mathrm{S} 4_{\mathrm{F}}^{2}$. This is clearly an equivalence relation. Write $|A|$ for the equivalence class determined by the formula $A \in$ Form $^{\square}$, and let $\mathbf{A}^{*}$ be the set of equivalence classes. We turn $\mathbf{A}^{*}$ into an interior algebra by defining $\Gamma^{*}, \sqcup^{*},-^{*}, 0^{*}, 1^{*}$, and $\mathbf{I}^{*}$ formula-wise as follows:

$$
\begin{aligned}
|A| \Pi^{*}|B| & =|A \& B| \\
|A| \sqcup^{*}|B| & =|A \vee B| \\
-^{*}|A| & =|\sim A| \\
0^{*} & =|\sim(p \rightarrow p)| \\
1^{*} & =|p \rightarrow p| \\
\mathbf{I}^{*}|A| & =|\square A| .
\end{aligned}
$$

It is routine to check that each of these is well-defined and that $\left\langle\mathbf{A}^{*}, \Gamma^{*}, \sqcup^{*},-^{*}, 0^{*}, 1^{*}, \mathbf{I}^{*}\right\rangle$ is indeed an interior algebra. Let $\leq^{*}$ be the partial order defined as above: $|A| \leq^{*}|B|$ iff $|A| \sqcup^{*}|B|=|B|$. Note:

- for any $A, B \in$ Form $^{\square},|A| \leq^{*}|B|$ iff $(A \rightarrow B) \in \mathrm{S} 4_{\mathrm{F}}^{2}$ and

- $1^{*}=\mathrm{S} 4_{\mathrm{F}}^{2}$.

Clearly, every finite subset of $\mathbf{A}^{*}$ has both a least upper bound and a greatest lower bound. But so do some infinite subsets. For any $A \in \operatorname{Form}^{\square}$ and $p \in \mathrm{PV}$, define $\mathbf{J}(A, p) \subseteq$ $\mathbf{A}^{*}$ as follows: $\mathbf{J}(A, p)=_{\mathrm{df}}\left\{\left|\iota_{p}^{B}(A)\right|: B \in \mathrm{F}\right\}$. We claim that $|\exists p A|=\operatorname{lu} b(\mathbf{J}(A, p))$ and $|\forall p A|=g l b(\mathbf{J}(A, p))$. We give the argument only for $|\exists p A|$. To see that $|\exists p A|$ is an upper bound of $\mathbf{J}(A, p)$, it suffices to note that, by Corollary $1.2,\left(l_{p}^{B}(A) \rightarrow \exists p A\right) \in \mathrm{S} 4_{\mathrm{F}}^{2}$, for

${ }^{4}$ Kit Fine and Saul Kripke independently discovered, but did not publish, proofs of this for the modal logics in the 1970s, though in [1], Fine provides an earlier proof sketch that second-order arithmetic can be recursively embedded into these modal logics. [4] provides published proofs for the modal results. [6] gives a proof for $\mathrm{H}$. It should be noted that [1] and [5] independently provide axiomatizations of the principal interpretation, in the Kripke semantics, of S5. 
every $B \in \mathrm{F}$. To see that $|\exists p A|$ is the least upper bound of $\mathbf{J}(A, p)$, suppose that $|C|$ is some upper bound of $\mathbf{J}(A, p)$. Choose a $q \in \mathrm{PV}$ that does not occur in either $A$ or $C$. Note that $\left|\imath_{p}^{q}(A)\right| \in \mathbf{J}(A, p)$ so that $\left|\imath_{p}^{q}(A)\right| \leq^{*}|C|$. Thus $\left(\imath_{p}^{q}(A) \rightarrow C\right) \in \mathrm{S} 4_{\mathrm{F}}^{2}$. So $\forall q\left(\imath_{p}^{q}(A) \rightarrow\right.$ $C) \in \mathrm{S} 4_{\mathrm{F}}^{2}$, since $\mathrm{S} 4_{\mathrm{F}}^{2}$ is closed under generalization. So $\left(\exists q l_{p}^{q}(A) \rightarrow C\right) \in \mathrm{S} 4_{\mathrm{F}}^{2}$, by Axiom Scheme 2 , since $q$ does not occur in $C$. Note that $\exists p A \stackrel{\text { alpha }}{=} \exists q \imath_{p}^{q}(A)$, since $q$ does not occur in $A$. So $(\exists p A \rightarrow C) \in \mathrm{S} 4_{\mathrm{F}}^{2}$, by Lemma 1.1. So $|\exists p A| \leq|C|$, as desired.

In general, if $\langle\mathbf{A}, \sqcap, \sqcup,-, 0,1, \mathbf{I}\rangle$ is an interior algebra, then a join is a subset of $\mathbf{A}$ that has a least upper bound, and a meet is a subset of $\mathbf{A}$ that has a greatest lower bound. Thus $\mathbf{J}(A, p)$ is both a join and a meet in $\mathbf{A}^{*}$.

If $\langle\mathbf{A}, \sqcap, \sqcup,-, 0,1, \mathbf{I}\rangle$ and $\left\langle\mathbf{A}^{\prime}, \Pi^{\prime}, \sqcup^{\prime},-^{\prime}, 0^{\prime}, 1^{\prime}, \mathbf{I}^{\prime}\right\rangle$ are interior algebras and $h: \mathbf{A} \rightarrow$ $\mathbf{A}^{\prime}$, then $h$ is a homomorphism iff, for every $a, b \in \mathbf{A}$, we have $h(a \sqcap b)=h(a) \sqcap^{\prime} h(b)$, $h(a \sqcup b)=h(a) \sqcup^{\prime} h(b), h(-a)=-^{\prime} h(a), h(0)=0^{\prime}, h(1)=1^{\prime}$, and $h(\mathbf{I} a)=\mathbf{I}^{\prime} h(a)$. If $h$ is a one-one homomorphism, then $h$ is an embedding. Suppose that $\mathcal{J} \subseteq \mathcal{P}(\mathbf{A})$ is a set of joins and $\mathcal{K} \subseteq \mathcal{P}(\mathbf{A})$ is a set of meets. An embedding $h$ is a $(\mathcal{J}, \mathcal{K})$-embedding iff for every $\mathbf{J} \in \mathcal{J}$ and every $\mathbf{K} \in \mathcal{K}$ we have the following: $\{h(a): a \in \mathbf{J}\}$ has a least upper bound in $\mathbf{A}^{\prime}$ and $\operatorname{lub}(\{h(a): a \in \mathbf{J}\})=h(\operatorname{lub}(\mathbf{J}))$; and $\{h(a): a \in \mathbf{K}\}$ has a greatest lower bound in $\mathbf{A}^{\prime}$ and $g l b(\{h(a): a \in \mathbf{J}\})=h(\operatorname{lu} b(\mathbf{J}))$.

The following lemma follows immediately from [11], Chapter III, Proposition 4.3, on page 101:

LEMMA 2.1. Suppose that $\mathbf{A}=\langle\mathbf{A}, \sqcap, \sqcup,-, 0,1, \mathbf{I}\rangle$ is a nondegenerate interior algebra, and that $\mathcal{J}$ and $\mathcal{K}$ are countable sets of joins and meets in $\mathbf{A}$, respectively. Then there is a topological space $X$ and $a(\mathcal{J}, \mathcal{K})$-embedding $h: \mathbf{A} \rightarrow \mathbf{A}_{X}$, where $\mathbf{A}_{X}$ is the algebra of subsets of $X$.

Given Lemma 2.1, we can now prove completeness. Let $\mathbf{A}^{*}$ be the Lindenbaum algebra for $\mathrm{S} 4{ }_{\mathrm{F}}^{2}$ as defined above. Let $\mathcal{J}=\left\{\mathbf{J}(A, p): A \in\right.$ Form $\left.^{\square} \& p \in \mathrm{PV}\right\}$. Since $|\exists p A|=$ $\operatorname{lub}(\mathbf{J}(A, p))$ and $|\forall p A|=\operatorname{glb}(\mathbf{J}(A, p))$, the set $\mathcal{J}$ is both a set of joins and a set of meets. Also note that $\mathcal{J}$ is countable, since $\mathbf{A}^{*}$ is countable, since there are only countably many formulas. By Lemma 2.1 , there is a topological space $X$ and a $(\mathcal{J}, \mathcal{J})$-embedding $h: \mathbf{A}^{*} \rightarrow \mathbf{A}_{X}$.

Consider the structure $\mathbf{S}^{*}=\langle X, D\rangle$, where $D$ is the image of the set $\{|A|: A \in \mathrm{F}\}$ under the $(\mathcal{J}, \mathcal{J})$-embedding $h$. For any $\varphi: \mathrm{PV} \rightarrow$ Form $^{\square}$, let $V^{\varphi}$ be the following valuation: $V^{\varphi}(p)=h(|\varphi(p)|)$. Note that every valuation on $\mathbf{S}^{*}$ is of this form, since $D$ is the image of $\{|A|: A \in \mathrm{F}\}$ under $h$. Also note, for future use, the following: $V^{\varphi_{p}^{C}}=V^{\varphi}[h(|C|) / p]$, for any $p \in \mathrm{PV}$ and $C \in \mathrm{F}$.

Now we will show the following, for every formula $A$ :

$$
\text { for every } \varphi: \mathrm{PV} \rightarrow \text { Form }^{\square}, V_{\text {mod }}^{\varphi}(A)=h(|\varphi(A)|) \text {. }
$$

We proceed by induction on $A$. If $A$ is atomic, the result is by the definition of $V^{\varphi}$. If $A=(B \& C)$, note that $V_{\text {mod }}^{\varphi}(A)=V_{\text {mod }}^{\varphi}(B \& C)=V_{\text {mod }}^{\varphi}(B) \cap V_{\text {mod }}^{\varphi}(C)=h(|\varphi(B)|) \cap$ $h(|\varphi(C)|)$ (by the inductive hypothesis) $=h\left(|\varphi(B)| \Pi^{*}|\varphi(C)|\right)$ (since $h$ is a homomorphism) $=h(|\varphi(B) \& \varphi(C)|)$ (by the definition of $\left.\sqcap^{*}\right)=h(|\varphi(B \& C)|)=h(|\varphi(A)|)$. The argument is the same if $A$ is of one of the forms $(B \vee C),(B \rightarrow C), \sim B$ or $\square B$. Suppose that $A=\exists p B$. Then note the following, where $q \in \mathrm{PV}$ is chosen so that $q$ does not occur in $B$ and is not free in $\varphi(r)$ for any propositional variable $r$ occurring in $B$ : 


$$
\begin{aligned}
h(|\varphi(A)|) & =h(|\varphi(\exists p B)|)=h\left(\left|\exists q \varphi_{p}^{q}(B)\right|\right)=h\left(\operatorname{lub}\left(\mathbf{J}\left(\varphi_{p}^{q}(B), q\right)\right)\right) \\
& =h\left(\operatorname{lub}\left(\left\{\left|l_{q}^{C}\left(\varphi_{p}^{q}(B)\right)\right|: C \in \mathrm{F}\right\}\right)\right) \\
& =h\left(\operatorname{lub}\left(\left\{\left|\varphi_{p}^{C}(B)\right|: C \in \mathrm{F}\right\}\right)\right) \text {, since } \imath_{q}^{C}\left(\varphi_{p}^{q}(B)\right) \stackrel{\text { alpha }}{=} \varphi_{p}^{C}(B) \\
& =\bigcup\left\{h\left(\left|\varphi_{p}^{C}(B)\right|\right): C \in \mathrm{F}\right\}, \text { since } h \text { is a }(\mathcal{J}, \mathcal{J}) \text {-embedding } \\
& =\bigcup\left\{V_{\bmod }^{\varphi_{p}^{C}}(B): C \in \mathrm{F}\right\}, \text { by the inductive hypothesis } \\
& =\bigcup\left\{V_{\bmod }^{\varphi}[h(|C|) / p](B): C \in \mathrm{F}\right\} \\
& =\bigcup\left\{V_{\bmod }^{\varphi}[P / p]_{\bmod }(B): P \in D\right\}, \text { since } D \text { is the image under } h \\
& \quad \text { of the set }\{|C|: C \in \mathrm{F}\} \\
& =V_{\bmod }^{\varphi}(\exists p B)=V_{\bmod }^{\varphi}(A), \text { as desired. }
\end{aligned}
$$

The argument in the case $A=\forall p B$ is similar.

Given $(*)$, we can draw two conclusions:

1. The structure $\mathbf{S}^{*}=\langle X, D\rangle$, is modally closed under $\mathrm{F}$. To see this, suppose that $V: \mathrm{PV} \rightarrow D$ and $A \in \mathrm{F}$. We want to show that $V_{\bmod }(A) \in D$. Recall that $D$ is the image of $\{|A|: A \in \mathrm{F}\}$ under $h$. So, for each $p \in \mathrm{PV}$, we can choose a $A_{p} \in \mathrm{F}$ so that $V(p)=h\left(\left|A_{p}\right|\right)$. Define $\varphi: \mathrm{PV} \rightarrow$ Form $\square$ as follows: $\varphi(p)=A_{p}$. Note that $V_{\text {mod }}=V_{\text {mod }}^{\varphi}$. So by $(*), V_{\text {mod }}(A)=h(|\varphi(A)|)$. Also, since $A \in \mathrm{F}$ and $\mathrm{F}$ is closed under substitutions (see page 510), $\varphi(A) \in \mathrm{F}$. So $h(|\varphi(A)|) \in D$. So $V_{\bmod }(A) \in D$, as desired.

2. $V_{\text {mod }}^{l}(A)=h(|l(A)|)=h(|A|)$, since $A \stackrel{\text { alpha }}{=} l(A)$.

Thus we get completeness for class of all structures modally closed under $\mathrm{F}$ as follows: suppose that $\mathbf{S} \vDash A$ for every structure $\mathbf{S}$ in this class. Then, in particular, $\mathbf{S}^{*} \vDash A$. Let $M$ be the model $\left\langle X, D, V^{\imath}\right\rangle$. Then $M \vDash A$. So $V_{\text {mod }}^{l}(A)=X$. So $h(|A|)=X$. So $|A|=1^{*}$ in the Lindenbaum algebra $\mathbf{A}^{*}$. So $A \in \mathrm{S} 4_{\mathrm{F}}^{2}$, as desired.

§3. Completeness of $\mathrm{H}_{\mathrm{F}}^{2}$. Fix a suitable $\mathrm{F}$. The completeness proof for $\mathrm{H}_{\mathrm{F}}^{2}$ is almost exactly the same as the completeness proof for $\mathrm{S}_{\mathrm{F}}^{2}$, except that this time we use Heyting algebras instead of interior algebras. A Heyting algebra is a seven-tuple $\mathbf{A}=$ $\langle\mathbf{A}, \sqcap, \sqcup, \Rightarrow,-, 0,1\rangle$, where $\mathbf{A}$ is a nonempty set; $\sqcap, \sqcup$, and $\Rightarrow$ are binary operations on $\mathbf{A}$; - is a unary operation on $\mathbf{A}$; and 0 and 1 are members of $\mathbf{A}$ all satisfying the following conditions, where $a \leq b$ is defined as $a \sqcup b=b$ :

- $\langle\mathbf{A}, \sqcap, \sqcup, 0,1\rangle$ is a bounded distributive lattice, that is,

- $\quad \sqcap$ and $\sqcup$ are commutative and associative;

- $a \sqcap(a \sqcup b)=a \sqcup(a \sqcap b)=a$, for every $a, b \in \mathbf{A}$;

- $\quad \sqcap$ and $\sqcup$ distribute over each other; and

- $a \sqcap 1=a$ and $a \sqcup 0=a$, for every $a \in \mathbf{A}$.

- $\quad a \Rightarrow b$ is the greatest element such that $a \sqcap(a \Rightarrow b) \leq b$.

- $(a \Rightarrow 0)=-a$, for every $a \in \mathbf{A}$. 
It is easy to see that $\leq$ is a partial order, and that $a \sqcap b$ and $a \sqcup b$ are the greatest lower bound and least upper bound, respectively, of $\{a, b\}$. $\mathbf{A}$ is degenerate if $0=1$. Otherwise $\mathbf{A}$ is nondegenerate.

One useful example is the algebra of open subsets of a topological space. If $X$ is a topological space, then define $\mathbf{A}_{X}={ }_{\mathrm{df}}\left\langle\mathcal{O}(X), \cap, \cup, \Rightarrow_{X}, \sim_{X}, \emptyset, X\right\rangle$. It is easy to check that this is a Heyting algebra.

Another useful example of a Heyting algebra is the Lindenbaum algebra for $\mathrm{H}_{\mathrm{F}}^{2}$. Say that any two formulas $A$ and $B$ are $\mathrm{H}_{\mathrm{F}}^{2}$-equivalent iff $(A \leftrightarrow B) \in \mathrm{H}_{\mathrm{F}}^{2}$. Write $|A|$ for the equivalence class determined by the formula $A \in$ Form, and let $\mathbf{A}^{*}$ be the set of equivalence classes. We turn $\mathbf{A}^{*}$ into a Heyting algebra by defining $\sqcap^{*}, \sqcup^{*}, \Rightarrow^{*},-^{*}, 0^{*}$, and $1^{*}$ formula-wise as follows:

$$
\begin{aligned}
|A| \sqcap^{*}|B| & =|A \& B| \\
|A| \sqcup^{*}|B| & =|A \vee B| \\
|A| \Rightarrow^{*}|B| & =|A \rightarrow B| \\
-^{*}|A| & =|\sim A| \\
0^{*} & =|\sim(p \rightarrow p)| \\
1^{*} & =|p \rightarrow p| .
\end{aligned}
$$

It is routine to check that each of these is well-defined, and that $\left\langle\mathbf{A}^{*}, \Pi^{*}, \sqcup^{*}, \Rightarrow^{*},-^{*}, 0^{*}, 1^{*}\right\rangle$ is indeed a Heyting algebra. Let $\leq^{*}$ be the partial order defined as above: $|A| \leq^{*}|B|$ iff $|A| \sqcup^{*}|B|=|B|$.

Just as in the modal case, we have $|\exists p A|=l u b(\mathbf{J}(A, p))$ and $|\forall p A|=g l b(\mathbf{J}(A, p))$, where $\mathbf{J}(A, p)=_{\mathrm{df}}\left\{l_{p}^{B}(A): B \in \mathrm{F}\right\}$. In general, if $\langle\mathbf{A}, \sqcap, \sqcup, \Rightarrow,-, 0,1\rangle$ is an Heyting algebra, then a join is a subset of $\mathbf{A}$ that has a least upper bound, and a meet is a subset of $\mathbf{A}$ that has a greatest lower bound. Thus $\mathbf{J}(A, p)$ is both a join and a meet in $\mathbf{A}^{*}$.

If $\langle\mathbf{A}, \sqcap, \sqcup, \Rightarrow,-, 0,1\rangle$ and $\left\langle\mathbf{A}^{\prime}, \Pi^{\prime}, \sqcup^{\prime}, \Rightarrow^{\prime},-^{\prime}, 0^{\prime}, 1^{\prime}\right\rangle$ are Heyting algebras and $h$ : $\mathbf{A} \rightarrow \mathbf{A}^{\prime}$, then $h$ is a homomorphism iff, for every $a, b \in \mathbf{A}$, we have $h(a \sqcap b)=$ $h(a) \Pi^{\prime} h(b), h(a \sqcup b)=h(a) \sqcup^{\prime} h(b), h(a \Rightarrow b)=h(a) \Rightarrow^{\prime} h(b), h(-a)=-^{\prime} h(a)$, $h(0)=0^{\prime}$, and $h(1)=1^{\prime}$. If $h$ is a one-one homomorphism, then $h$ is an embedding.

Suppose that $h$ is an embedding of $\langle\mathbf{A}, \sqcap, \sqcup, \Rightarrow,-, 0,1\rangle$ in $\mathbf{A}_{X}=\left\langle\mathcal{O}(X), \cap, \cup, \Rightarrow_{X}\right.$, $\left.\sim_{X}, \varnothing, X\right\rangle$ for some topological space $X$. Also suppose that $\mathcal{J} \subseteq \mathcal{P}(\mathbf{A})$ is a set of joins. We say that $h$ is a $\mathcal{J}$-embedding iff for every $\mathbf{J} \in \mathcal{J}$ and every meet $\mathbf{K} \subseteq \mathbf{A}$, we have

- $h(\operatorname{lub}(\mathbf{J}))=\bigcup\{h(a): a \in \mathbf{J}\}$ and

- $h(g l b(\mathbf{K}))=\operatorname{Int}_{X}(\bigcap\{h(a): a \in \mathbf{K}\})$.

The following lemma follows immediately from [11], Chapter IV, Proposition 9.2, on page 140:

Lemma 3.1. Suppose that $\mathbf{A}=\langle\mathbf{A}, \sqcap, \sqcup, \Rightarrow,-, 0,1\rangle$ is a nondegenerate Heyting algebra, and that $\mathcal{J}$ is a countable sets of joins in $\mathbf{A}$. Then there is a topological space $X$ and $a \mathcal{J}$-embedding $h: \mathbf{A} \rightarrow \mathbf{A}_{X}$.

Given Lemma 3.1, the proof of completeness proceeds as in the modal case. Let $\mathbf{A}^{*}$ be the Lindenbaum algebra for $\mathrm{H}_{\mathrm{F}}^{2}$. Let $\mathcal{J}=\{\mathbf{J}(A, p): A \in$ Form \& $p \in \mathrm{PV}\}$, which is a countable set of joins. By Lemma 3.1, there is a topological space $X$ and a $\mathcal{J}$-embedding $h: \mathbf{A}^{*} \rightarrow \mathbf{A}_{X}$. Consider the structure $\mathbf{S}^{*}=\langle X, D\rangle$, where $D$ is the image of the set $\{|A|: A \in \mathrm{F}\}$ under $h$. For any $\varphi:$ PV $\rightarrow$ Form, let $V^{\varphi}$ be the following 
valuation: $V^{\varphi}(p)=h(|\varphi(p)|)$. As in the modal case, we can show the following, for every formula $A$;

$$
\text { for every } \varphi: \mathrm{PV} \rightarrow \text { Form, } V_{\text {int }}^{\varphi}(A)=h(|\varphi(A)|) .
$$

And given $(*)$, we can prove completeness just as in the modal case.

§4. The Barcan and constant-domain schemes. Consider the following two axiom schemes, the Barcan scheme in the language $\mathcal{L}^{\square}$ and the constant-domain scheme in $\mathcal{L}$ :

$$
\begin{aligned}
& \text { B } \quad \forall p \square A \rightarrow \square \forall p A \\
& \text { C } \forall p(A \vee B) \rightarrow(A \vee \forall p B)(p \text { is not free in } A) .
\end{aligned}
$$

And consider any $\mathrm{F} \subseteq$ Form $\square[\mathrm{F} \subseteq$ Form $]$, suitable or not. Let $\mathrm{S} 4_{\mathrm{F} \mathbf{B}}^{2}\left[\mathrm{H}_{\mathrm{FC}}^{2}\right]$ be the smallest set of formulas containing every theorem of $\mathrm{S}_{\mathrm{F}}^{2}\left[\mathrm{H}_{\mathrm{F}}^{2}\right]$, every instance of $\mathbf{B}[\mathbf{C}]$, and closed under modus ponens, generalization and, in the modal case, necessitation.

Theorems 4.1 and 4.2, below, are from the 1970s: they are completeness results for certain of the $\mathrm{S}_{\mathrm{FB}}^{2}$ and $\mathrm{H}_{\mathrm{FC}}^{2}$, for the Kripke semantics. Theorems 4.3 and 4.4, below, are analogous results for the topological semantics. As we shall see, the topological results are immediate corollaries to the Kripke results.

Say that a Kripke frame is an ordered pair $K=\langle W, R\rangle$, where $W$ is a nonempty set and $R$ is a reflexive and transitive relation on $W$. We define a set $\mathcal{U}(K) \subseteq \mathcal{P}(W)$, of up-sets of $K=\langle W, R\rangle$ as follows: $P \in \mathcal{U}(K)$ iff $P \subseteq W$ and $\forall w \in P, \forall w^{\prime} \in W$, if $w R w^{\prime}$ then $w^{\prime} \in P$. Take a (constant-domain) Kripke structure to be an ordered triple $\langle W, R, D\rangle$ where $\langle W, R\rangle$ is a Kripke frame and $D \subseteq \mathcal{P}(W)$. If $K=\langle W, R\rangle$, then the Kripke structure $\langle W, R, D\rangle$ is intuitionistic iff $D \subseteq \mathcal{U}(K)$. Given a Kripke structure $\langle W, R, D\rangle$, a valuation on $\langle W, R, D\rangle$ is a function $V: \mathrm{PV} \rightarrow D$. A Kripke model is an ordered quartuple $M=$ $\langle W, R, D, V\rangle$, where $\langle W, R, D\rangle$ is a Kripke structure and $V$ is a valuation on $\langle W, R, D\rangle$. A Kripke model $\langle W, R, D, V\rangle$ is intuitionistic iff $\langle W, R, D\rangle$ is intuitionistic.

As in the topological semantics, given a Kripke structure $\langle W, R, D\rangle$, we can extend every valuation $V$ to two functions $V_{\text {mod }}:$ Form $^{\square} \rightarrow \mathcal{P}(W)$, which gives the modal value of each formula in Form ${ }^{\square}$; and $V_{\text {int }}$ : Form $\rightarrow \mathcal{U}(K)$, which gives the intuitionistic value of each formula in Form. The clauses for $\&, \vee, \rightarrow, \sim$, and $\square$ are as in standard modal and intuitionistic logic and the clauses for $\forall$ and $\exists$ in the modal and intuitionistic case are as follows (with the subscript 'mod' or 'int' suppressed):

$$
\begin{aligned}
& V(\forall p A)=\bigcap_{P \in D} V[P / p] A \\
& V(\exists p A)=\bigcup_{P \in D} V[P / p] A .
\end{aligned}
$$

The definitions of soundness and completeness are analogous to the topological case. Ditto for the definition of when a Kripke structure [intuitionistic Kripke structure] is modally [intuitionistically] closed under a set $F$ of formulas. The 1970s results for Kripke semantics are as follows: ${ }^{5}$

THEOREM 4.1 (Fine [1], 1970). If $\mathrm{F}=\mathrm{F}_{\square \forall \exists}$ or Form ${ }^{\square}$, then $\mathrm{S}_{\mathrm{FB}}^{2}$ is sound and complete for the class of constant-domain Kripke structures that are closed under F.

5 The axiomatizations of $\mathrm{S} 4_{\mathrm{FB}}^{2}$ and $\mathrm{H}_{\mathrm{FC}}^{2}$ in the cited literature are a bit different from, but equivalent to, ours. 
THEOREM 4.2 (Gabbay [2], 1974, generalized in Sobolev [12], 1977). If $\mathrm{PV} \subseteq \mathrm{F}$, then $\mathrm{H}_{\mathrm{FC}}^{2}$ is sound and complete for the class of intuitionistic constant-domain Kripke structures that are closed under $\mathrm{F}$.

The analogous topological results follow from the fact that we can treat any Kripke frame as a topological space. More precisely, suppose that $K=\langle W, R\rangle$ is a Kripke frame. Then we get a topological space if we start with the underlying set $W$ and take the open sets to be the up-sets. ${ }^{6}$ Moreover suppose that $K=\langle W, R\rangle$ is a Kripke frame, that $\langle W, R, D\rangle$ is a constant-domain Kripke structure, and that $\langle W, R, D, V\rangle$ is a constant-domain Kripke model. Consider the Alexandroff space $X$ whose underlying set is $W$ and whose open sets are the up-sets in $K$. Note that $\langle X, D\rangle$ is a topological structure (and is intuitionistic if $\langle W, R, D\rangle$ is intuitionistic) and that $\langle X, D, V\rangle$ is a topological model. Moreover, if we calculate the functions $V_{\text {mod }}$ and $V_{\text {int }}$ using the topological semantics, then we get the same values as we would get using the Kripke semantics. Thus, the following results follow immediately from Theorems 4.1 and 4.2 , above.

THEOREM 4.3. If $\mathrm{F}=\mathrm{F}_{\square \forall \ngtr 7}$ or Form $\square$, then $\mathrm{S}_{\mathrm{FB}}^{2}$ is sound and complete for the class of Alexandroff topological structures that are closed under $\mathrm{F}$.

THEOREM 4.4. If $\mathrm{PV} \subseteq \mathrm{F}$, then $\mathrm{H}_{\mathrm{FC}}^{2}$ is sound and complete for the class of Alexandroff topological structures that are closed under $\mathrm{F}$.

\$5. Appendix. On page 511, we promised an Appendix giving intrinsic conditions on a topological structure equivalent to that structure being closed under Form ${ }^{\square}$. By "intrinsic conditions", we mean conditions that do not mention formulas.

Consider any topological structure $\langle X, D\rangle$. We will consider $n$-place functions on $D$, where $n \in \mathbb{N}$, including $n=0$ : the zero-place functions on $D$ will simply be members of $D$. More specifically, we will define a family of finite-place functions on $D$, which we will call the formula-like functions. Then we will observe that $\langle X, D\rangle$ is closed under Form $\square$ iff $D$ is closed under every formula-like function.

Given any $m, n \in \mathbb{N}$ with $m<n$, and $n$-place functions $f$ and $g$ on $D$, we define new finite-place functions $\operatorname{not}_{n}(\mathrm{f}), \operatorname{and}_{n}(\mathrm{f}, \mathrm{g}), \operatorname{or}_{n}(\mathrm{f}, \mathrm{g}), \operatorname{arrow}_{n}(\mathrm{f}, \mathrm{g}), \operatorname{box}_{n}(\mathrm{f}, \mathrm{g}), \operatorname{all}_{n}^{m}(\mathrm{f})$, and some $_{n}^{m}(\mathrm{f})$, on $D$ as follows:

$$
\begin{aligned}
\operatorname{not}_{n}(\mathrm{f})\left(P_{1}, \ldots, P_{n}\right) & =X-\mathrm{f}\left(P_{1}, \ldots, P_{n}\right) \\
\operatorname{and}_{n}(\mathrm{f}, \mathrm{g})\left(P_{1}, \ldots, P_{n}\right) & =\mathrm{f}\left(P_{1}, \ldots, P_{n}\right) \cap \mathrm{g}\left(P_{1}, \ldots, P_{n}\right) \\
\operatorname{or}_{n}(\mathrm{f}, \mathrm{g})\left(P_{1}, \ldots, P_{n}\right) & =\mathrm{f}\left(P_{1}, \ldots, P_{n}\right) \cup \mathrm{g}\left(P_{1}, \ldots, P_{n}\right) \\
\operatorname{arrow}_{n}(\mathrm{f}, \mathrm{g})\left(P_{1}, \ldots, P_{n}\right) & =\left(X-\mathrm{f}\left(P_{1}, \ldots, P_{n}\right)\right) \cup \mathrm{g}\left(P_{1}, \ldots, P_{n}\right) \\
\operatorname{box}_{n}(\mathrm{f})\left(P_{1}, \ldots, P_{n}\right) & =\operatorname{Int}\left(\mathrm{f}\left(P_{1}, \ldots, P_{n}\right)\right) \\
\operatorname{all}_{n}^{m}(\mathrm{f})\left(P_{1}, \ldots, P_{n-1}\right) & =\bigcap_{P \in D} \mathrm{f}\left(P_{1}, \ldots, P_{m-1}, P, P_{m+1}, \ldots, P_{n-1}\right) \\
\operatorname{some}_{n}^{m}(\mathrm{f})\left(P_{1}, \ldots, P_{n-1}\right) & =\bigcup_{P \in D} \mathrm{f}\left(P_{1}, \ldots, P_{m-1}, P, P_{m+1}, \ldots, P_{n-1}\right) .
\end{aligned}
$$

${ }^{6}$ Incidentally, the resulting space is an Alexandroff space, i.e., arbitrary intersections of open sets are open. 
Given any $n \geq 1$, any $m \leq n$, we define an $n$-place projection function $\operatorname{proj}_{n}^{m}$ on $D$ :

$$
\operatorname{proj}_{n}^{m}\left(P_{1}, \ldots, P_{n}\right)=P_{m} .
$$

Finally, the family of formula-like functions on $D$ is the smallest set of functions that contains all of the projection functions and that is closed under all of the not $_{n}$, and ${ }_{n}$, or $_{n}$, arrow $_{n}$, box ${ }_{n}$, allm ${ }_{n}^{m}$, and some ${ }_{n}^{m}$. Observe that $\langle X, D\rangle$ is closed under Form ${ }^{\square}$ iff $D$ is closed under every formula-like function.

\title{
BIBLIOGRAPHY
}

[1] Fine, K. (1970). Propositional quantifiers in modal logic. Theoria, 36, 336-346.

[2] Gabbay, D. (1974). On 2nd order intuitionistic propositional calculus with full comprehension. Archiv f ur mathematische Logik, 16, 177-186.

[3] Geuvers, H. (1994). Consevativity between logics and typed lambda-calculi. In Barendregt, H. and Nipkow, T., editors. Types for Proofs and Programs, International Workshop TYPES '93. Lecture Notes in Computer Science, Vol. 806. New York: Springer, pp. 131-154.

[4] Kaminski, M. \& Tiomkin, M. (1996). The expressive power of second-order propositional modal logic. Notre Dame Journal of Formal Logic, 37, 35-43.

[5] Kaplan, D. (1970). S5 with quantifiable propositional variables. Journal of Symbolic Logic, 35, 355.

[6] Kremer, P. (1997a). On the complexity of propositional quantification in intuitionistic logic. Journal of Symbolic Logic, 62, 529-544.

[7] Kremer, P. (1997b). Propositional quantification in the topological semantics for S4. Notre Dame Journal of Formal Logic, 38, 295-313.

[8] Kremer, P. (2013). Strong completeness of S4 for any dense-in-itself metric space. Review of Symbolic Logic, 6, 545-570.

[9] Kremer, P. (2014). Quantified modal logic on the rational line. Review of Symbolic Logic, 7, 439-454.

[10] Makinson, D. (1966). On some completeness theorems in modal logic. Zeitschrift für mathematische Logik und Grundlagen der Mathematik, 12, 379-384.

[11] Rasiowa, H. \& Sikorski, R. (1963). The Mathematics of Metamathematics. Warsaw: Państowowe Wydawnictwo Naukowe,

[12] Sobolev, S. K. (1977). The intuitionistic propositional calculus with quantifiers (in Russian). Matematicheskiye Zametki, 22, 69-76. English translation in Mathematical Notes of the Academy of Sciences of the USSR 22 (1977), 528-532.

[13] Sørensen, M. H. \& Urzyczyn, P. (2006). Lectures on the Curry-Howard Isomorphism. New York: Elsevier.

[14] Zdanowski, K. (2009). On second order intuitionistic propositional logic without a universal quantifier. Journal of Symbolic Logic, 74, 157-167.

\author{
DEPARTMENT OF PHILOSOPHY \\ UNIVERSITY OF TORONTO \\ TORONTO, ON M5S 3H7, CANADA \\ E-mail:kremer@utsc.utoronto.ca
}

